BULL. AUSTRAL. MATH. SOC.

\title{
A NOTE ON SPACES RELATED TO NAMIOKA SPACES
}

\author{
J.P. Lee and Z. Piotrowski
}

\begin{abstract}
Namioka proved that the following condition (*) given below holds, if $X$ is Čech-complete and $Y$ is a locally compact, $\sigma$-compact space.
\end{abstract}

(*) Let $X$ and $Y$ be spaces, $Z$ be a metric space and let $f: X \times Y \rightarrow Z$ be separately continuous. Then there is a dense, $G_{\delta}$ set $A$ in $X$ such that $A \times Y \subset C(f)$.

Following Christensen a space $X$ is called Namioka if (*) is true for any compact space $Y$. In this paper we introduce and study a new class of spaces which is closely related to Namioka spaces. Namely, we say that a space $Y$ is co-Namioka if (*) holds for any Namioka space $X$.

\section{Introduction}

There are many papers which deal with the classical problem of determining the points of continuity for a separately continuous function;

Received 18 october 1984. The authors would like to thank their colleagues, R.A. McCoy and D.J. Lutzer for their helpful remarks; they. also thank J.B. Brown who permitted them to use his unpublished example (see Example 2).

The second-named author wishes to extend his thanks to the Department of Mathematics of the State University of New York at Old Westbury for inviting him to the Summer Workshop on Topology, which made this paper possible.

Copyright Clearance Centre, Inc. Serial-fee code: 0004-9727/85 $\$ A 2.00+0.00$. 
see for example [2], [4], [10] or [11].

Throughout this paper, a space means a completely regular topological one. The set of points of continuity of $f$ will be denoted by $C(f)$.

In what follows, a general condition given below and denoted by (*), will be called a continuity statement.

(*) Let $X$ and $Y$ be spaces, $Z$ be a metric space and let $f: X \times Y \rightarrow Z$ be separately continuous. Then there is a dense, $G_{\delta}$ set $A$ in $X$ such that $A \times Y \subset C(f)$.

Namioka [10] proved that $\left(^{*}\right)$ holds if $X$ is strongly countable complete (Čech-complete) and $Y$ is locally compact and $\sigma$-compact.

A space $X$ is Namioka [3] if (*) is true for any compact space $Y$.

So all Čech-complete spaces are Namioka [10] see also [2] and [11], where it is proved that some spaces defined by topological games are Namioka.

LEMMA 1 ([5]). Let $X$ be a Baire space.

(a) If $A_{1}, A_{2}, \ldots, A_{n}, \ldots$ are dense, $G_{\delta}^{\prime} s$ of $X$, then so is

$\infty$

$\prod_{i=1}^{\infty} A_{i} \cdot($ Theorem 10.1).

(b) A subset of $X$ which is the complement of a first category set contains a dense, $G_{\delta}$ subset of $X$ (Exercise 6, p. 281).

LEMMA 2 ([11], Theorem 3, p. 501). Namioka spaces are Baire.

\section{Co-Namioka spaces}

In the main results of [2] and [11] (which are of type (*)), the space $Y$ is assumed to be compact. So the following question arises.

What is a class $W$ of spaces, strictly larger than the class $C$ of all compact spaces, such that $\left(^{*}\right)$ is true for any Namioka space $X$ and $Y \in W ?$

Let $S$ be a "nice" subclass of Namioka spaces, for example LC - the class of all locally compact spaces.

A space $Y$ will be called co-Namioka (respectively co-Namioka rel $S$ ) 
if (*) holds for any Namioka space $X$ (respectively any space $X$ from S).

Obviously, by this definition, compact spaces are co-Namioka; furthermore, co-Namioka spaces are co-Namioka spaces rel $S$, for any subclass of Namioka spaces $N$.

The following Proposition 1 was proved in [10] in case of strongly countably complete spaces $X$. Our proof uses the method of Namioka and relies heavily on Lemma 2.

PROPOSITION 1. Every locally compact o-compact space is co-Namioka.

Proof. Since $y$ is locally compact and o-compact there is a sequence $\left\{y_{i}: i=1,2, \ldots\right\}$ of compact subsets of $Y$ such that

$Y=\bigcup_{i=1}^{\infty}$ Int $Y_{i}$ ([5], Theorem 7.2, p. 241). Since $X$ is Namioka, for every $i$, there is a dense $G_{\delta}$ set $A_{i}$ in $X$ such that $f \mid X \times Y$ is continuous on $A_{i} \times Y_{i}$. But then, clearly, $f$ is continuous at each point of $\left(\bigcap_{i=1}^{\infty} A_{i}\right) \times y$. Now by Lemma $1(a), A=\bigcap_{i=1}^{\infty} A_{i}$ is a dense, $G_{\delta}$ subset of $X$, the latter being Namioka, and hence, by Lemma 2, Baire. So there is a dense, $G_{\delta}$ set $A \subset X$, with $A \times Y \subset C(f)$.

Proposition 1 suggests the question:

Must all co-Namioka spaces be Baire?

The following proposition that follows from [4], Theorem 2, p. 647, Lemma $I(b)$ and Lemma 2, answers this question in the negative.

PROPOSITION 2. Every second countable space is co-Namioka.

This means, in particular, that if $X$ is the unit interval and $Y$ is the set $Q$ of rational numbers, then (*) holds (!). Recall that Christensen [3], Theorem 1, p. 114, showed that if $X$ is the set $Q$ of rational numbers, $Y$ is the unit interval and $z=C_{p}\left(Q^{2},[-1,1]\right)$, the space of continuous functions from $Q^{2}$ into $[-1,1]$ equipped with the pointwise topology $\left(c_{p}\left(Q^{2},[-1,1]\right)\right.$ is compact metric $)$, then there is a 
separately continuous $f: X \times Y \rightarrow Z$ which does not satisfy (*).

Similarly, as in Proposition 2, it can be shown, using [13], Theorem 2, p. 438, Lemma 1 (b) and Lemma 2, that if we assume, additionally, that the range $Z$ of separately continuous functions considered in (*) is a compact space, then every first countable space is co-Namioka.

In an attempt to generalize simultaneously both Propositions 1 and 2 , say to all Lindelöf spaces (recall that in the class of locally compact spaces, a space is $\sigma$-compact if and only if it is Lindelöf [5], Theorem 7.2 , p. 241) the following example ([12], Remark (b), p. 241) arises.

EXAMPLE 1. There is a hereditarily Lindelöf and hereditarily separable space which is not co-Namioka.

Proof. Let $X$ and $Z$ be the unit interval $I$ and let $Y$ be the space $C_{p}(I, I)$ of continuous functions from $I$ into $I$ equipped with the pointwise topology. Then $f(x, y)=y(x)$ is the required function.

The fact that $y$ is hereditarily Lindelöf and hereditarily separable, easily follows from the fact that $I$ has a countable network. It can be shown that $C_{p}(I, I)$ is of first category in itself [7], and is not a Frechét space and thus not first countable; this follows since $C_{p}(I, R)$ can be embedded in $C_{p}(I, I)$ and since $C_{p}(I, R)$ is not a k-space ([6] and $[8])$.

So, if not all Lindelöf, even hereditarily Lindelöf, spaces are coNamioka, then perhaps either all locally compact spaces that are also paracompact or all $k$-spaces are co-Namioka? Again, the answer is no, even if we assume that such a space is both complete and metric.

The following unpublished example, due to Brown, was originally designed to answer Christensen's question [2] whether (*) holds for complete metric spaces $X$ and $Y$.

EXAMPLE 2. There is a complete metric, locally compact space which is not co-Namioka.

Proof. Let $X=[0,1], Y=\underset{\alpha \in[0,1]}{\dot{U}} Y_{\alpha}, Y_{\alpha}=[0,1]$ and let $Z=R$. 
The set $X \times Y$, equipped in "the open-page-book topology", is the free union of compact squares ("pages") $X \times Y_{\alpha}$, with $\alpha \in[0,1]$.

Now let us order them in "a long line" and let us define, for every $\alpha \in[0,1]$, a separately, but not jointly, continuous function $f: X \times Y_{\alpha} \rightarrow Z$, requiring though, that the point (or points) of discontinuity of $f_{\alpha}$ is (respectively are spread out) somewhere in $\{\alpha\} \times y_{\alpha}$

Now it is easy to see that if $f(x, y) \stackrel{\text { def }}{=} f_{\alpha}(x, y)$ for $(x, y) \in X \times y_{\alpha}$, then $f$ does not satisfy $(*)$. Hence $Y$ is not coNamioka, because $X$ is Namioka.

We now arrive at the main problem of the paper.

PROBLEM 1. Characterize co-Namioka spaces.

Let us recall that a partial answer to this problem was obtained by Talagrand and is the main result of [12], Theorem 3.1, p. 241.

TALAGRAND'S THEOREM. If $X$ is compact and $Y$ is a special K-analytic space, then (*) holds.

(In our terminology: Special K-analytic spaces are co-Namioka rel $\mathcal{C}$, where $\mathrm{C}$ stands for the class of compact spaces.)

The following problem is closely related to Talagrand's result and our theorem.

PROBLEM 2. Do co-Namioka and co-Namioka rel(C) spaces coincide?

We shall prove the following:

THEOREM. If $X$ is locally compact and $Y$ is a $k_{\omega}$-space, then (*) holds.

A space $X$ is called a $k_{\omega}$-space if $X=\bigcup_{n=I}^{\infty} X_{n}$ with $X_{n}$ compact and increasing and if $X$ has the weak topology of $X_{n}{ }^{\prime}$ 's; then this sequence $X_{1}, X_{2}, \ldots$ is called a $k_{\omega}$-decomposition of $X \cdot k_{\omega}$-spaces, as $\sigma$-compact spaces are Lindelöf and paracompact; moreover, they are 
precisely hemicompact $k$-spaces.

The set $Q$ of rational numbers, with the usual topology is not a $k_{\omega}$-space, although the same set $Q$ with the Sorgenfrey topology is $k_{\omega}$. The first example of a non-Baire $k_{\omega}$-space is due to Archangel'skit and Franklin [1].

Both special $K$-analytic and $k_{\omega}$-spaces contain, as Lindelöf spaces, those spaces that are both $\sigma$-compact and locally compact. However, the relation between special $K$-analytic and $k_{\omega}$-spaces is not completely understood.

In the proof of our theorem we rely on the following Lemma 3 ; the result shown in the lemma seems to be a part of folklore, however, we decided to attach a short, hopefully, new proof; compare [5], Proof of Theorem $4.4, \mathrm{p} .263$.

LEMMA 3. Let $X$ be locally compact and let $Y$ be a $k_{\omega}$-space. Then the product topology of $X \times Y$ coincides with the weak topology of the sets $X \times Y_{n}$, where the $Y_{n}$ 's are elements of some $k_{\omega}$ decomposition of $Y$.

Proof. The space $X$, a locally compact space, admits the Alexandroff compactification $\alpha X$, and $X$ is open in $\alpha X$. Therefore, every $X \times Y_{n}$ is open in $\alpha X \times Y_{n}$, for $n=1,2, \ldots$.

Next, let $U$ be a subset of $X \times Y$ that intersects every $X \times Y_{n}$ as an open set.

Then, for every $n$, the set $\left(\alpha X \times y_{n}\right) \cap U$ is open in $\alpha X \times y_{n}$. Hence we get that $U$ is open in $\alpha X \times Y$. Obviously, $U$ is then open in $X \times Y$. This finishes the proof of Lemma 3 .

REMARK. Since locally compact spaces are characterized as open subspaces of their Stone-Čech compactifications, [5], Theorem 8.3, p. 245, we see no way of extending Lemma 3 with its present proof.

Proof of the theorem. Clearly, we are able to determine the points of continuity of $f$ restricted to (countably many) "layers" $X \times Y_{n}$, 
$n=1,2, \ldots$. Now because the product topology of $X \times Y$ coincides with the weak topology of $X \times Y_{n}$ (Lemma 3) we can find such a simultaneous set, in $X$, whose Cartesian product with the space $Y$ is contained in $C(f)$. In other words, we apply Lemma 3 to any $k_{\omega}$-decomposition, which is a countable family of compact subsets of $Y$.

In fact, $f$ is continuous on $A_{n} \times Y_{n}$, where $A_{n}$ is a dense, $G_{\delta}$ (in $X$ ) and $y_{n}$ is a (compact) element of $k_{\omega}$-decomposition. Obviously, $f$ is continuous on $\left(\bigcap_{n=1}^{\infty} A_{n}\right) \times Y$ and $A=\prod_{n=1}^{\infty} A_{n}$ is a dense, $G_{\delta}, X$ being locally compact. This proves our theorem.

Obviously, the countability of the family $\left\{A_{n}\right\}$ is needed to get a simultaneous dense, $G_{\delta}$ set of points of continuity.

Similar arguments to those given in the proof of our theorem show the following result that is closely related to Mirzoian's theorem [9].

PROPOSITION 3. Let $X$ be locally compact, $Y$ be a metric, $k_{\omega^{-}}$ space, $Z$ be compact metric and let a function $f: X \times Y \rightarrow Z$ have all its $x$-sections $f_{x}$ continuous and its $y$-sections $f_{y}$ continuous, for the $y^{\prime} s$ belonging to a dense subset $D$ of $Y$. Then ere is a residual set $A \subset X$ such that $A \times Y \subset C(f)$.

\section{References}

[1] A.V. Archangel'skiY and S.P. Franklin, "Ordinal invariants for topological spaces", Michigan Math. J. 15 (1968), 313-320.

[2] J.P.R. Christensen, "Joint continuity of separately continuous functions", Proc. Amer. Math. Soc. 82 (1981), 455-461.

[3] J.P.R. Christensen, "Remarks on Namioka spaces and R.E. Johnson's theorem on the norm separability of the range of certain mappings", Math. Scand. 52 (1983), 11.2-116.

[4] J. Calbrix et J.P. Troallic, "Applications séparément continues", $C$. R. Acad. Sci. Paris, Sér. A 288 (1979), 647-648. 
[5] James Dirgundji, Topology (Allyn and Bacon, Boston, 1978).

[6] J. Gerlits, "Some properties of $C(X)$, II", Topology Appl. 15 $(1983), 255-262$.

[1] D.J. Lutzer and R.A. McCoy, "Category in function spaces", Pacific $J$. Math. 90 (1980), 145-168.

[8] R.A. McCoy, "k-space function spaces", Internat. J. Math. Math. Sci. 3 (1980), 701-711.

[9] M.M. Mirzoian, "On the cluster sets of mappings of topological spaces", Soviet Math. Dokl. 19 (1978), 1326-1329.

[10] 1. Namioka, "Separate and joint continuity", Pacific J. Math. 51 $(1974), 515-531$.

[11] J. Saint Raymond, "Jeux topologiques et espaces de Namioka", Proc. Amer. Math. Soc. 87 (1983), 499-504.

[12] M. Talagrand, "Deux generalisations d'un theoreme de I. Namioka", Pacific J. Math. 81 (1979), 239-251.

[13] J.D. Weston, "Some theorems on cluster sets", J. London Math. Soc. 33 (1958), 435-441.

Department of Mathematics,

State University of New York/College at Old Westbury,

Box 210 ,

Old Westbury,

Long Island,

New York II568,

USA;

Department of Mathematical and Computer Sciences,

Youngstown State University,

Youngstown,

Onio 44555,

USA. 